\title{
Brain Bridge: A Comparative Study between Database Querying and Human Memory Querying.
}

\author{
Suhas Suresh ${ }^{1}$, Pavan Kumar B S ${ }^{2}$, Aishwarya $\mathrm{U}^{3}$ \\ ${ }^{1}$ (Computer Science and Engineering, $R$ L Jalappa Institute of Technology, Bangalore, India) \\ ${ }^{2}$ (Computer Science and Engineering, Nagarjuna College of Engineering and Technology, Bangalore, India) \\ ${ }^{3}$ (Computer Science and Engineering, Sri Venkateshwara College of Engineering, Bangalore, India)
}

\begin{abstract}
The human memory is always been subject to many innovations and research since a very long time. As far as our knowledge exceeds, it is the fastest information retrieval system present. Further, the database management systems are specially designed applications that interact with the user to retrieve and analyze data. Its working can be best understood with the help of a human memory structure. While the database system itself is conceptualized from the human memory, it is important to know the similarities and working principles between these two. In this paper, we throw some light on the context of database structure, it working and we refer it with respect to the human memory system.
\end{abstract}

Keywords: Brain mapping, database system, human memory, indexing, and information retrieval.

\section{INTRODUCTION}

In an article published in The New York Times, dated September 24, 1991, it stated as follows, "After decades of research, scientists who study the brain are at last poised to explain how the brain is organized to form long term, conscious memory and later to recall a particular memory and its various components". This serves as the main theme of our paper. Further stating, it is shown in detail, as to how the human memory is understood with the help of a little example. In the same words, "In a paper published in the current Science, they describe the anatomical components of the brain's conscious memory system and suggest how it operates in connecting the many separate storage sites throughout the cortex that together represent a whole memory. The organ known as the hippocampus, they suggest, links the separate parts of a memory as it is formed, so enabling all to be evoked when the memory is recalled", thus, showing us the basic working of it.

Following is the review on how our paper would flow. In the human memory system, an event is cached back upon a basic principle. It takes the input from the external sensory information (sight, taste, touch, hearing, smell) and it checks for the corresponding keyword in the Referral Memory. Using that as an index, it searches for the main event in the Long Term Memory. Keeping this concept at the back of our minds, it is easier to explain the working of the database systems. When an input is given, it searches the corresponding keyword in the index table and thus, obtaining the relevant information from the secondary storage. While this is the basic working of any database model, it can be understood with the human memory working's perspective.

\section{HUMAN MEMORY WORKING}

In a recent article on Memory Retrieval by Richard C Mohs, he stated that we retrieve the information at an unconscious level but bring it to our conscious at our own will. While most of the people think, they have "good" or "bad" memory; it is an assumption that many people are good or bad at remembering things. But actually, if anyone has a problem in remembering things, then it must be due to a physical disease rather than any other difficulty.

Consider an example of placing a pair of sunglasses before we go to sleep. Before we go to bed, we must register where we had placed our glasses, pay attention while placing them on any table, and this information must be retained in order to retrieve it on a later time. The next morning, when we need to take the glass, we go to the exact table and collect it back as we had placed it. What if we forget where we had kept the glasses? The following can be the reasons for not being able to remember properly:

2.1 We may not have registered clearly where we put them down to begin with.

2.2 We may not have retained what we registered.

2.3 We may not be able to retrieve the memory accurately.

So in order to stop forgetting where we had left our sunglasses, we must take proper steps to make sure we store the information properly. If we have forgotten something, it may be due to not being able to encode it properly, because we were distracted while we were trying to encode it, or because we may have trouble retrieving it. If we've forgotten where we'd kept our glasses, we may not have forgotten. Rather we would have 
forgotten the place where we had kept them. This is nothing but the illustration of importance of memory indexing. And this is the main concern of our paper.

Another example which everyone can associate or relate to is-remembering an almost forgotten event after a very long time. It so happens that, when we come across a bite of a cookie after decades or smell of a perfume after a long time, we take time to recall what it is about and our association with it. After a short span of recalling, we may come up with the actual events associated with it. Here, the taste, smell, touch, sound, etc acts as an index to refer the long lost memory and its details. And the time taken to re-collect the events is nothing but the traditional searching techniques that are employed everywhere. While linear search is most time consuming, having a time complexity of $\mathbf{O}(\mathbf{n})$, it is rather discarded for being less efficient and more timeconsuming. However, the searching technique used in human memory system is super-efficient yet unknown and a mystery till date.

\section{WORKING OF DATABASE}

In general a database is an organized collection of data. A database management system is an application which allows the user to define, create, query, update, and administrate database. The database has two main and important functions-- how the data is stored in the memory, and how that data can be retrieved from it. Now let's go on with the working of the database.

Firstly let's see how data is stored. Whenever an input (data) is given to the database, it processes the input and it generates a key also known as index key. This index key will be stored in the index table; here index table is the primary search table, it acts just like a cache memory in computer system. After the key is generated, the whole data is stored in the data table. This storage may be in the form of stack, heap or linked list depending on the algorithm defined by the user.

Now let's go on with how data can be retrieved from the database. When a user wants to retrieve the data which he has previously stored, he queries the database which is the input. Now the database triggers the index table to search for the key. Once the key is obtained, keeping this key as reference it searches the data table for the relevant data. This search is carried out using search algorithms. Few search algorithms are linear search, binary search and so on. The time taken for this search is the processing time. After all this steps, the retrieved data is sent back to the ser as output.

Conceder an example - the database of a college. This database contains fields like name, class, marks of each subject. When we give this as an input the name acts as the key and is stored in the index table. And the other fields along with the name are stored in the data table. This is how data is stored. To retrieve the data we have to just give name as the input. This name will be searched in the index table first. After the key is found, it directly refers to the related data in the data table. Now this gives all the details related to that name which was previously stored. Fig. 1 illustrates the working of the database.

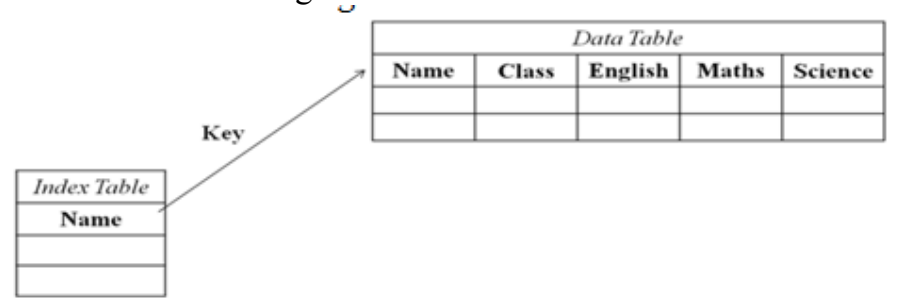

Fig.1: Basic structure of database.

\section{COMPARISON BETWEEN BRAIN AND DATABASE}

There is a clash going on in every database and the same clash in every human brain. The clash is simple memory verses CPU. Human brain either relay on indexes to recall the data or it counts on the CPU to calculate the resulting data.

The human brain works the same way as the database, the problem is Database Administrators and Developers alike constantly strive to increase the performance of the database. But humans do not make specific efforts to strike the same balance within the inner tables of the brain database. Whenever an input is given to the database, it processes the input and it generates a key called the index key. This key will be stored in the index table, here the index table is the primary search table, it acts like a cache memory in the computer system. After the key is generated the whole data is stored in the data table.

The human brain and database retrieve information using the index key. The human memory system takes input from the external sensory information (sight, taste, touch, and hearing) and it checks for the keyword in the referral memory and intern the keyword is used as an index and searched in the long term memory to retrieve the information. But in database the user queries the database which is the input and the database triggers the index table to search for the key, once the key is found it keeping the key as reference it searches the relevant data. The retrieved data is sent to the user as output. 
An example shows the working of brain indexing and database indexing. In brain indexing, when a person doesn't know the words of a certain song until someone starts the song, then suddenly the person continues to sing every word, that person started the song as a keyword which was searched in the brain. Hence the person doesn't have to remember the entire song but he has to remember the index which is searched in the memory. Sometimes if the person loses the index of the song, then the entire song is gone. In database indexing, it is similar to a phone book. In the front it tells you where in this massive book to find people in a certain city or where to find only those with the last name that starts with Y or Z. Database do the same thing, internally the index is stored in the memory for quick retrieval when a query for data retrieval is executed. Hence when someone searches the database with an index the information is quickly retrieved. From the above examples we can say that database indexing is much reliable than brain indexing as there are possibilities of losing data in brain indexing if the index is lost.

\section{IMPLEMENTATION}

In this context, we mainly consider the way our brain identifies human faces. It should be noted that along with faces, our brain can also very well recognize fragrance, smells, sense of touch, heat or cold, among others. For better understanding and easy implementation, we consider faces here.

Our brain has a big data set. It may consist of faces, events, places, numbers, etc. Let us consider a few faces from a large data set present in a human brain.

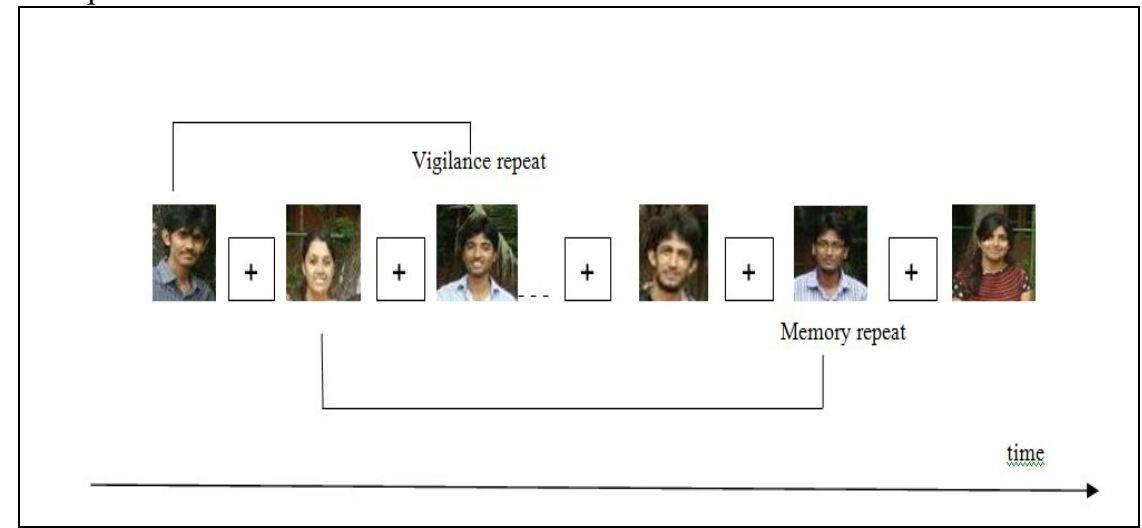

Fig 2. An illustration of face identification from a large data set.

In the figure 2, a participant is made to identify faces from a given data set. A noteworthy point here is that - the brain functions more accurately in querying a face database than any other database. Here, we examine interimage variability, its relativity and association of images with certain events, etc. In the Vigilance Repeat, the brain observes the first and third images thoroughly for querying. In the Memory Repeat, the brain repeats the same querying activity until it deduces a relationship between the second image and the fifth image. Once a relationship is establishes, corresponding activities that are chained/outlined to form a bountiful array of events. Based upon the data present at the back of the brain, relevant output can be expected. The output might be an association of that image with certain events. It can either be the first time they met or the assignment that any had done together or a project/ paper that they had worked upon or classes they had attended, etc. It should, however be noted that not all images can yield a positive result. Some associativity (read: data) may have gotten erased as the passage of time. A positive result is named as Hit Rate and False Alarm Rate. Hit rate is the positive outcome of a query while False Alarm Rate is the irrelevant or negative outcome of the querying. False alarm rate is the output when an image (or a face) is identical to another image. Given below is figure 3, a graph which shows the hit rate and false alarm rate for the data set that we had taken in Figure 2.

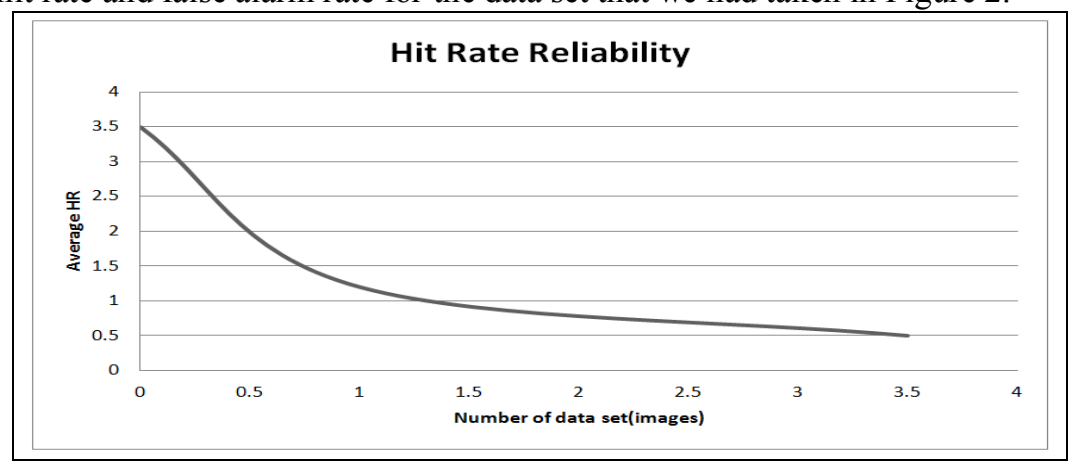

Figure 3 a). Hit Rate Reliability graph 


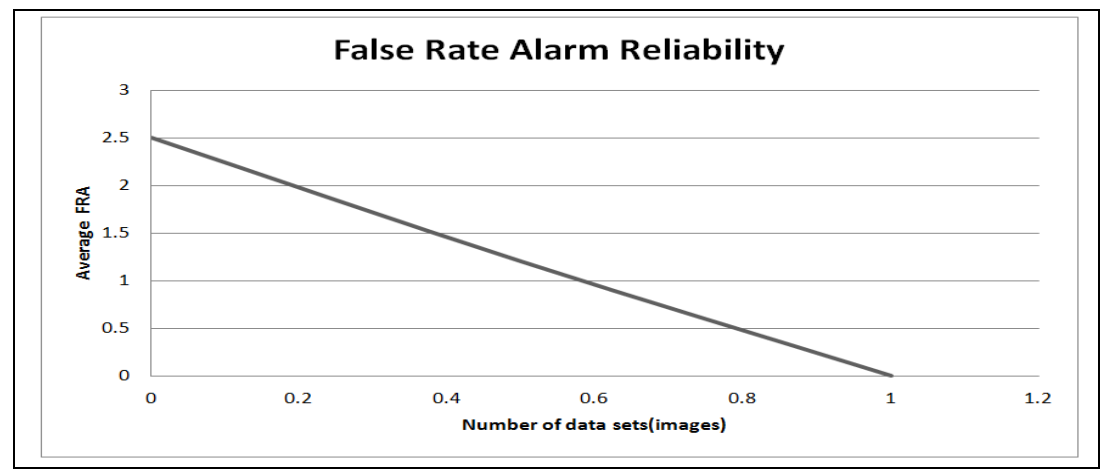

Figure 3 b). False Alarm Rate Reliability graph.

How does Face identification relate to RDBMS? : in the above figure and the consecutive explanation, it can be found that the image acts as an index that would lead us to a large data forum associated with it. Similarly, in RDBMS, we make use of an index, using which we can access a specific or rather general data set. And this is the main theme behind this paper.

\section{CONCLUSION}

This study serves as an initial, empirical look at the comparison between human brain and database systems. It draws parallel comparison between the traditional data base querying method and the human memory querying. We have observed that both have a few things in common. Few of them that are worth mentioning are - both have indexing mechanisms, both uses a vast set of data as their backdrop, etc. While it is impossible to illustrate senses as an index in this paper, we have tried to implement faces (images) that serve as an index in human memory querying. We have also obtained the hit rate and false alarm rate graphs for the data set that we have utilized. This paper also heralds the time efficiency of brain querying as compared to the database querying. This research opens the door to future investigation in various fields, from cognitive psychology to cognitive neuroscience to computer vision, as to what makes some face images, facial features or different senses more memorable than others ${ }^{11}$.

\section{REFERENCES}

[1] Bainbridge W., Isola P., Blank I., \& Oliva, A. (2012). Establishing a Database for Studying Human Face Photograph Memory. Poster presented at the 34thAnnual Conference of the Cognitive Science Society. Sapporo, Japan

[2] Brady, T. F., Konkle, T., Alvarez, G. A., \& Oliva, A. (2008). Visual long-term memory has a massive storage capacity for object details. Proceedings of the National Academy of Sciences, 105(38), 14325-14329

[3]. Philip J Hilts, A Brain unit Seen As Index For Recalling Memories, September 24, 1991, New York Times

[4]. Hans Moravec, When Will Computer Harware Match the human Brain, Journal of Evolution and Technology, 1998, Volume-1. 